MODELING, IDENTIFICATION AND CONTROL, 1990, vOL. 11, NO. 3, 169-179

doi:10.4173/mic.1990.3.4

\title{
Adaptive feedback linearization of non-linear systems with significant actuator dynamics
}

\author{
THOR I. FOSSEN
}

Keywords: Actuator dynamics, non-linear control, Lyapunov stability theory, adaptative feedback linearization, nonlinear decoupling.

\begin{abstract}
When applying feedback linearization techniques actuator dynamics is usually neglected. This is crucial for actuators with large time constants. A large class of systems, e.g. chemical processes, belong to this category. In such cases conventional feedback linearization techniques based on adaptive control or sliding control perform poorly. This paper discusses an approach which allows the inclusion of the actuator dynamics in the controller design. The design methodology has validation both for adaptive feedback linearization as well as sliding control. An augmented Lyapunov-like function candidate including the actuator states was used to prove global stability. This is done by applying Barbalat's Lyapunov-like lemma for nonautonomous systems. The control law is simulated for both the nominal and adaptive cases. In the adaptive case both the plant parameters and the actuator time constant are assumed to be unknown.
\end{abstract}

\section{Introduction}

Feedback linearization techniques allow the designer to compensate for nonlinearities in a plant when designing the control law. Controllers based on feedback linearization have high performance and may be used for a large number of operating conditions. The major problem with feedback linearization techniques is robustness due to imprecise cancellations of model non-linearities and neglected actuator dynamics. In the case of parametric uncertainties, global asymptotically stable controllers may be found by using Lyapunov stability theory. Two useful results from the stability theory are adaptive feedback linearization and sliding control. Both approaches have been successfully applied in robotic control for several years, Slotine and $\mathrm{Li}$ (1987). Sliding control has also been applied in the control of underwater vehicles which are highly non-linear and time-varying in their parameters, Yoerger and Slotine (1985). This paper shows how adaptive feedback linearization can be applied to non-linear systems with significant actuator dynamics. Such systems frequently appear in process control as well as in the control of mechanical systems.

\section{Nominal design}

In nominal design we will assume perfect knowledge of both the plant dynamics and the actuator dynamics. The adaptive case will be discussed in the next section.

Received 22 July 1990.

Division of Engineering Cybernetics, Norwegian Institute of Technology, N 7034 Trondheim, Norway. 
2.1. Review of input-output feedback linearization

We briefly review some of the results from Sastry and Isidori (1989) and Slotine and Li (1991). Consider an SISO non-linear minimum phase system described as:

$$
x^{(n)}=f(X)+b(X) u
$$

where $X=\left[x, \dot{x}, \ldots, x^{(n-1)}\right]^{\mathrm{T}}$ is a measurable state vector and the scalar $u$ is the control input. This control problem may be reduced to that of controlling the linear system:

$$
x^{(n)}=v_{x}
$$

A stable closed loop system is obtained for $v_{x}$ defined as:

$$
v_{x}=x^{(n)}-\left(\frac{d}{d t}+\lambda_{x}\right)^{n} \tilde{x}
$$

where $\tilde{x}=x-x_{d}$ is the tracking error, $x_{d}$ is the desired state vector and $\lambda_{x}$ is a positive constant which may be interpreted as the control bandwidth. If $b(X)$ in Eqn. 1 is nonsingular for all $\boldsymbol{X}$, the actual control input $u$ is calculated as:

$$
u=\frac{1}{b(X)}\left(v_{x}-f(X)\right)
$$

Slotine and Li (1987) define a sliding surface:

$$
s=\left(\frac{d}{d t}+\lambda_{x}\right)^{(n-1)} \tilde{x}
$$

The relationship between $v_{x}$ and $s$ is simply:

$$
\dot{s}+\lambda_{x} s=x^{(n)}-v_{x}=0
$$

This ensures that the sliding surface $s \rightarrow 0$ and thus $\tilde{x} \rightarrow 0$. For cases $n=1$ and $n=2$ these expressions are simply:

$$
\begin{array}{lll}
n=1, & s=\tilde{x} & v_{x}=\dot{x}_{d}-\lambda_{x} \tilde{x} \\
n=2, & s=\hat{x}+\lambda_{x} \tilde{x} & v_{x}=\ddot{x}_{d}-2 \lambda_{x} \hat{x}-\lambda_{x}^{2} \tilde{x}
\end{array}
$$

Let $\alpha>0$ be the reference model's eigenvalues and let $r_{x}$ be the commanded input. This suggests the reference model can be chosen as:

$$
\begin{array}{ll}
n=1, & \dot{x}_{d}+\alpha x_{d}=\alpha r_{x} \\
n=2, & \ddot{x}_{d}+2 \alpha \dot{x}_{d}+\alpha^{2} x_{d}=\alpha^{2} r_{x}
\end{array}
$$

or in general:

$$
\left(\frac{d}{d t}+\alpha\right)^{n} x_{d}=\alpha^{n} r_{x}
$$

\subsection{Actuator dynamics}

Consider a non-linear SISO system in the form:

$$
\begin{aligned}
& \dot{x}=f(x)+b(x) u \\
& \dot{u}=-\frac{1}{T}\left(u-u^{\prime}\right)
\end{aligned}
$$


where $T$ is the actuator time constant and $u^{\prime}$ is the actuator input. To apply the results from the previous section, we must first calculate $\ddot{x}$ :

$$
\ddot{x}=\left(\frac{\partial f(x)}{\partial x}+\frac{\partial b(x)}{\partial x} u\right) \dot{x}+b(x) \dot{u}=\left(\frac{\partial f(x)}{\partial x}+\frac{\partial b(x)}{\partial x} u\right)(f(x)+b(x) u)-\frac{b(x)}{T}\left(u-u^{\prime}\right)
$$

which suggests the non-linear control law:

$$
u^{\prime}=\frac{T}{b(x)}\left[v_{x}-\left(\frac{\partial f(x)}{\partial x}+\frac{\partial b(x)}{\partial x} u\right)(f(x)+b(x) u)\right]+u
$$

A major disadvantage with this approach is that we have to calculate $\partial f(x) / \partial x$ and $\partial b(x) / \partial x$. In the adaptive case, we also have to measure $\dot{x}$ which is quite restrictive. This suggests a new scheme. By splitting the design into two parts:(1) design of an inner loop and (2) design of an outer loop, a much simpler decoupling strategy is found.

\section{Design of inner loop}

Again, assume that the actuator dynamics is as described in Eqn. 9. If we define the desired behaviour of the actuator dynamics as:

$$
\dot{u}_{d}+\lambda_{u} u_{d}=\lambda_{u} r_{u}
$$

where $\lambda_{u}$ is a positive constant and $r_{u}$ is an unknown commanded input will be interpreted later. Define a Lyapunov-like function candidate:

$$
V_{u}(\tilde{u}, t)=\frac{1}{2} \tilde{u}^{2} \text { where } \tilde{u}=u-u_{d}
$$

Selecting the control law:

$$
u^{\prime}=T v_{u}+u
$$

with $v_{u}$ defined as:

$$
v_{u d}=\dot{u}_{d}-\lambda_{u} \tilde{u}
$$

yields the error equation:

$$
\tilde{u}+\lambda_{u} \tilde{u}=0
$$

and thus:

$$
\dot{V}_{u}=-\lambda_{u} \tilde{u}^{2} \leqslant 0
$$

According to Barbalat's Lyapunov-like lemma this ensures that the inner loop is globally asymptotically stable, i.e. $u$ converges to $u_{d}$ in finite time for all initial values. Eliminating $\dot{u}$ from Eqns. 9 and 16 and substituting Eqn. 12 yields the following expression for $r_{u}$.

$$
r_{u}=\left(1-\frac{1}{T \lambda_{u}}\right) u+\frac{1}{T \lambda_{u}} u^{\prime}
$$

\section{Design of outer loop}

Let the controller be chosen as Eqn. 14 with $v_{u}$ defined as in Eqn. 15. The commanded input $r_{u}$ in the reference model Eqn. 12 is found from the following assumption: 
Assumption 1: High bandwidth inner loop

In the design of the inner loop, $\lambda_{u}$ is assumed to be selected such that:

$$
T \lambda_{u} \gg 1
$$

This implies that Eqn. 18 simplifies to:

$$
r_{u} \approx u
$$

which suggests the inner loop commanded input $r_{u}$ to be selected as Eqn. 4, namely:

$$
r_{u}=\frac{1}{b(X)}\left(v_{x}-f(X)\right)
$$

This particular choice of $r_{u}$ implies that the closed loop system consists of a high bandwidth inner loop with bandwidth $\lambda_{u}$ decoupling the actuator dynamics and an outer loop with bandwidth $\lambda_{x}$ decoupling the plant dynamics, see Fig. 1 .

Define a Lyapunov-like function candidate:

$$
V_{x}(s, t)=\frac{1}{2} s^{2}
$$

where $s$ is defined in Eqn. 5. Differentiating $V_{x}$ with respect to time yields:

$$
\dot{V}_{x}=s \dot{s}=s\left(f(X)+b(X) u-v_{x}-\lambda_{x} s\right)
$$

From assumption 1, we have: $u=r_{u}$ which implies that:

$$
\dot{V}_{x}=-\lambda_{x} s^{2} \leqslant 0
$$

which again according to Barbalat's lemma ensures that $s$ and $\tilde{x}$ converge to zero.

\section{Adaptive feedback linearization}

Adaptive feedback linearization is described in detail by Slotine and Coetsee (1986) and Slotine and $\mathrm{Li}(1987,1991)$. To generalize these results for the case where we have actuator dynamics, let us consider a system in the form:

$$
\begin{aligned}
x^{(n)} & =f(X)+b u \\
\dot{u} & =-\frac{1}{T}\left(u-u^{\prime}\right)
\end{aligned}
$$

where $b$ is an unknown constant with known sign, $f$ is a nonlinear function with unknown constant or slowly varying parameters and $T$ is a positive unknown time constant. To derive a control law that ensures perfect tracking in spite of parametric uncertainties, we select the control law as:

$$
u^{\prime}=\hat{T} v_{u}+u
$$

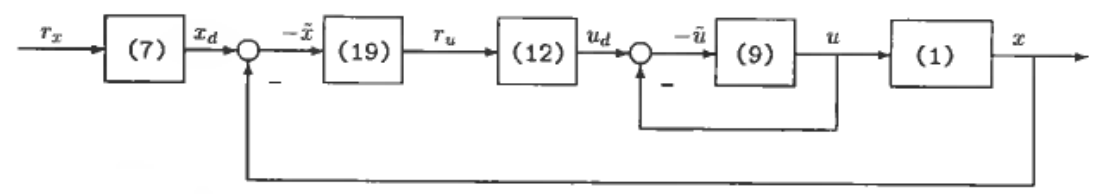

Figure 1. Closed loop system dynamics. The numbers in brackets denote the equation numbers. 
where the hat denotes the adaptive estimate and $v_{x}$ and $v_{u}$ are defined as in the nominal case. Assume that $f(\boldsymbol{X})$ is linear in its parameters $\theta$, such that $f(\boldsymbol{X})$ may be written as:

$$
f(X)=\varphi^{\mathrm{T}}\left(X, v_{x}\right) \theta
$$

where $\varphi\left(X, v_{x}\right)$ is a non-linear known regression vector. Combining Eqns. 22 and 23 yields:

$$
\begin{aligned}
& \dot{s}+\lambda_{x} s=x^{(n)}-v_{x}=b\left[-\left(\frac{\hat{f}}{\bar{b}}-\frac{f}{b}\right)+\left(\frac{1}{\bar{b}}-\frac{1}{b}\right) v_{x}\right]=b \varphi^{\mathrm{T}}\left(X, v_{x}\right) \tilde{\theta} \\
& \hat{u}+\lambda_{u} \tilde{u}=\dot{u}-v_{u}=\frac{1}{T} \tilde{T} v_{u}, T>0
\end{aligned}
$$

where, $\tilde{\boldsymbol{\theta}}=\hat{\boldsymbol{\theta}}-\boldsymbol{\theta}$ is the parameter error vector and $\tilde{T}=\hat{T}-T$ is the time constant error. In the adaptive case the inner loop commanded input $r_{u}$ is found as:

$$
r_{u}=\left(1-\frac{1}{T \lambda_{u}}\right) u+\frac{1}{T \lambda_{u}} u^{\prime}-\frac{\widetilde{T}}{T \lambda_{u}} v_{u}
$$

where the last term is due to the uncertainties in the actuator time constant. Again, we assume that $\lambda_{u}$ can be selected such that $T \lambda_{u} \gg 1$ which implies that $r_{u} \approx u$. This suggests that the commanded input $r_{u}$ is to be selected as:

$$
r_{u}=\frac{1}{b(X)}\left(v_{x}-\hat{f}(X)\right)
$$

To derive the parameter update laws, we propose a Lyapunov-like function candidate:

$$
V(s, \tilde{u}, t)=\frac{1}{2}\left(s^{2}+\tilde{u}^{2}+\frac{\beta}{T} \tilde{T}^{2}+|b| \tilde{\theta}^{\mathrm{T}} \Gamma \tilde{\theta}\right), \quad T>0
$$

where, $\beta$ is a positive constant and $\Gamma$ is a positive matrix of appropriate dimension, usually diagonal. The following adaption laws:

lead to:

$$
\begin{aligned}
& \tilde{\theta}=-\operatorname{sgn}(b) \Gamma^{-1} \varphi^{T}\left(X, v_{x}\right) s \\
& \tilde{T}=-\frac{1}{\beta} v_{u} \tilde{u}
\end{aligned}
$$

$$
\dot{V}=-\lambda_{x} s^{2}-\lambda_{u} \tilde{u}^{2} \leqslant 0
$$

According to Barbalat's lemma this ensures that the sliding surface $s$ converges to zero and thus $\tilde{x}$ converges to zero. It is important to notice that the parameter estimates will be bounded. To implement the controller we must replace the parameter update laws with: $\dot{\hat{\boldsymbol{\theta}}}=\hat{\boldsymbol{\theta}}$ and $\tilde{\hat{T}}=\hat{T}$. This is based on the assumption that the true plant parameters are constant or at least slowly varying, i.e. $\dot{\theta}=0$ and $\dot{T}=0$.

\section{Case study}

\subsection{Simplified model of a marine vehicle}

The motion of a marine vehicle in surge may be described as:

$$
\begin{aligned}
& \dot{x}=-a x|x|+b u \\
& \dot{u}=-\frac{1}{T}\left(u-u^{\prime}\right)
\end{aligned}
$$


where $a, b$ and $T$ are unknown constant parameters. Defining the decoupling parameters $v_{x}$ and $v_{u}$ as:

$$
\begin{aligned}
& v_{x}=\dot{x}_{d}-\lambda_{x} \tilde{x} \\
& v_{u}=\dot{u}_{d}-\lambda_{u} \tilde{u}
\end{aligned}
$$

the control law becomes:

$$
u^{\prime}=\hat{T} v_{u}+u
$$

Defining the parameters $\theta_{1}=\frac{a}{b}$ and $\theta_{2}=\frac{1}{b}$, the desired states $u_{d}, x_{d}$ are calculated from:

$$
\begin{aligned}
& r_{u}=\varphi^{\mathrm{T}} \hat{\theta}=\hat{\theta}_{1} x|x|+\hat{\theta}_{2} v_{x} \\
& \dot{u}_{d}+\lambda_{u} u_{d}=\lambda_{u} r_{u} \\
& \dot{x}_{d}+\alpha x_{d}=\alpha r_{x}
\end{aligned}
$$

Global stability is guaranteed if the parameter update laws are chosen as:

$$
\begin{aligned}
& \hat{\theta}_{1}=-\frac{1}{\gamma_{1}} x|x| s \\
& \hat{\theta}_{2}=-\frac{1}{\gamma_{2}} v_{x} s \\
& \hat{T}=-\frac{1}{\beta} v_{u} \tilde{u}
\end{aligned}
$$

where the sliding surface $s$ is simply $s=\tilde{x}$.

\subsection{Simulation result}

In the first simulation we assumed that the plant and actuator parameters were perfectly known, i.e. $a=0.5, b=10$, and $T$ was varied as: $T=0.1 \mathrm{~s}$ (Fig. 2$), T=1 \mathrm{~s}($ Fig. 3) and $T=10$ s (Fig. 4). Each simulation was performed for two different cases:

Case 1: Decoupling of both the actuator (inner loop) and the plant (outer loop) according to the scheme presented in Section 2.

Case 2: Decoupling of the plant (outer loop) only, but the actuator dynamics (inner loop) is still included in the simulations.

The sampling rate used in the simulations was $10 \mathrm{~Hz}$ while the control bandwidths were selected as: $\lambda_{u}=10$ and $\lambda_{x}=1$. The commanded input $r_{x}$ was switched between $r_{x}=0-3$ and $r_{x}=0-1$. For $T=0-1 s$ the tracking error $\tilde{x}$, the plant input $u$ and the actuator input $u^{\prime}$ were practically equal for Cases 1 and 2. Fig. 2 illustrates the time responses for both cases. When we increase the actuator time constant $T$ by a factor of 10 , the results are quite different, Fig. 3. In Case 1, the tracking error is quite similar to that of Fig. 2. For the case where we only decouple the plant, we notice a significant increase in the tracking error. This trend is clearer for $T=10 \mathrm{~s}$, Fig. 4. Here the performance is quite unsatisfactory for Case 2, while Case 1 still yields good performance. The simulation results for the nominal case illustrate that the non-linear decoupling should include the actuator dynamics when the time constant for the actuator dynamics exceeds approximately $0-1 \mathrm{~s}$. For other systems this limit may of course vary. 

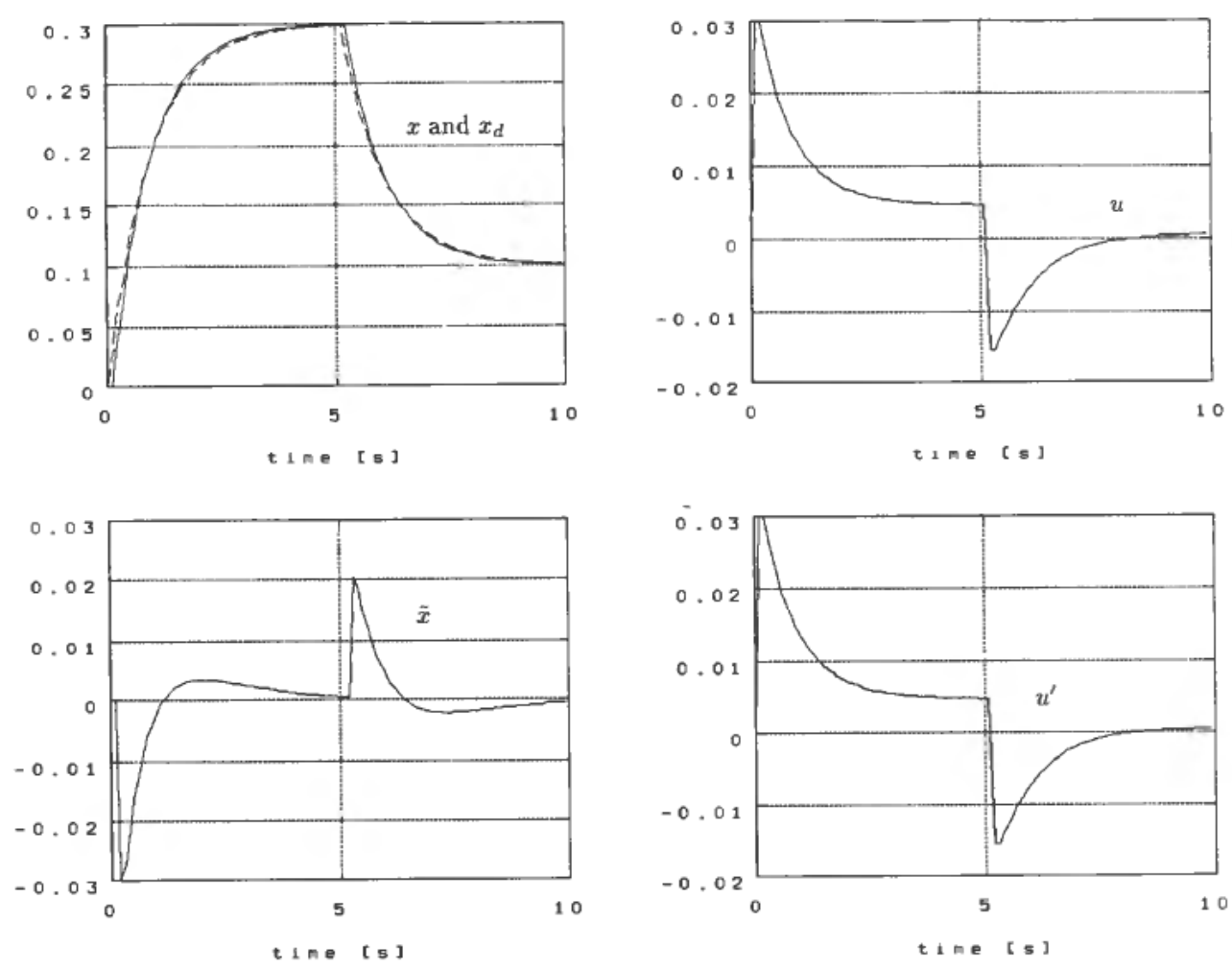

Figure 2. Nominal design $T=0.1 \mathrm{~s}$. 


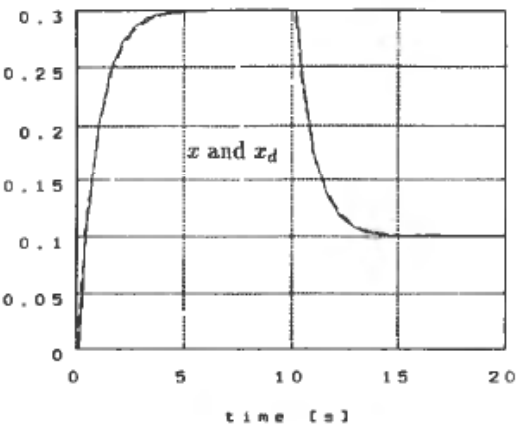

Case 1
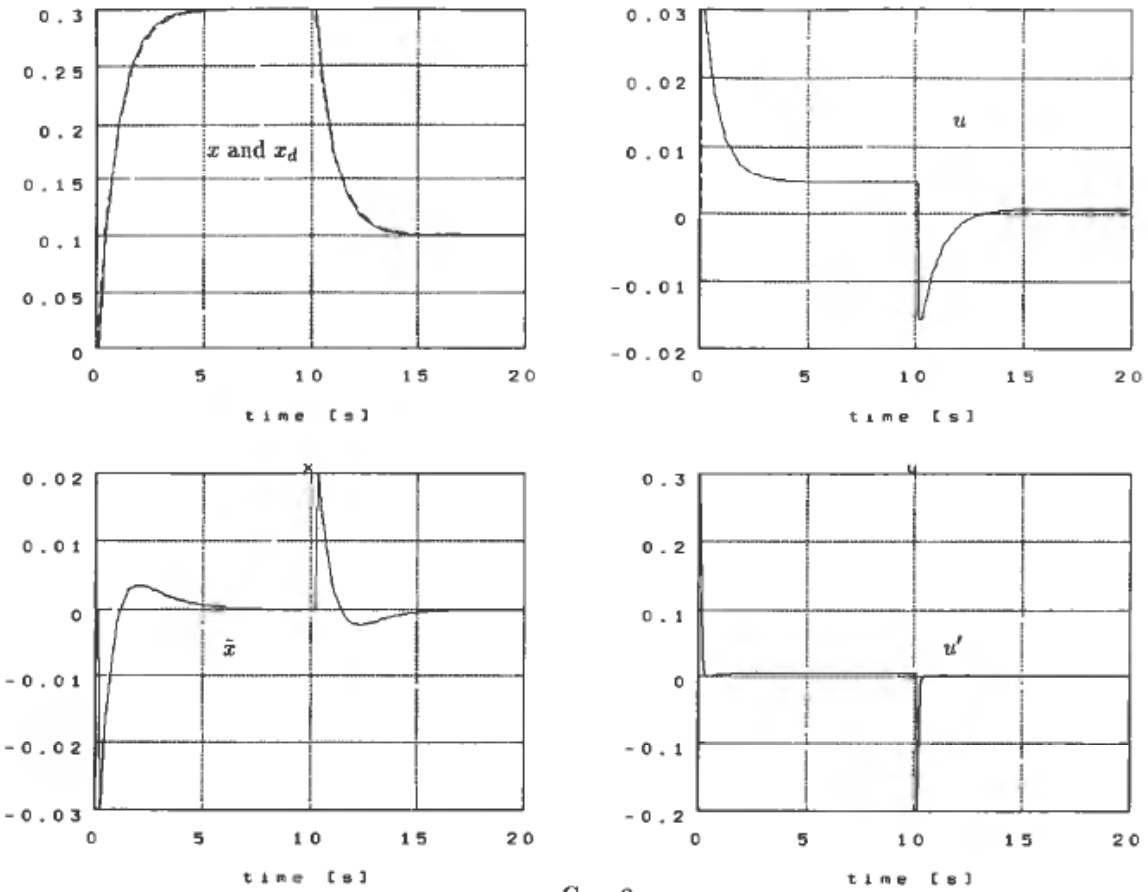
Case 2
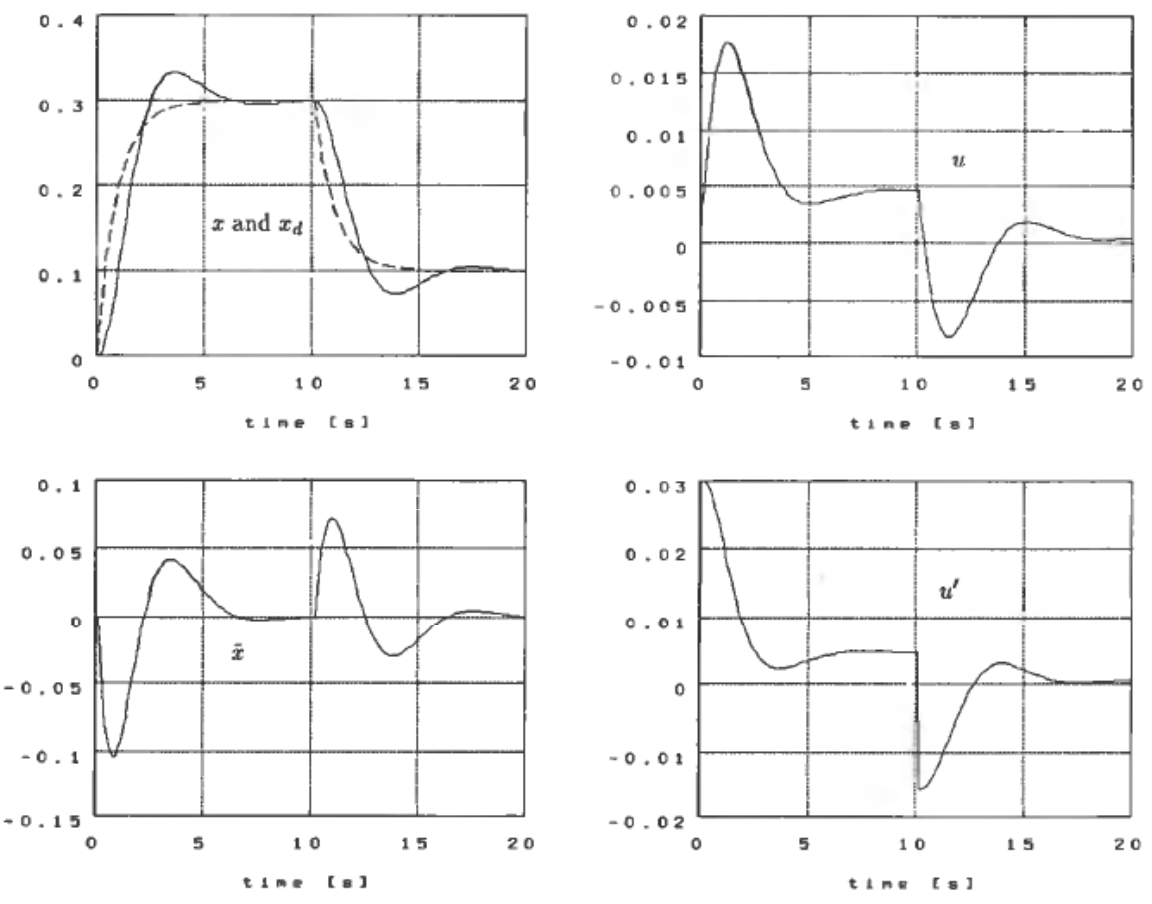

Figure 3. Nominal design $T=1 \mathrm{~s}$. 

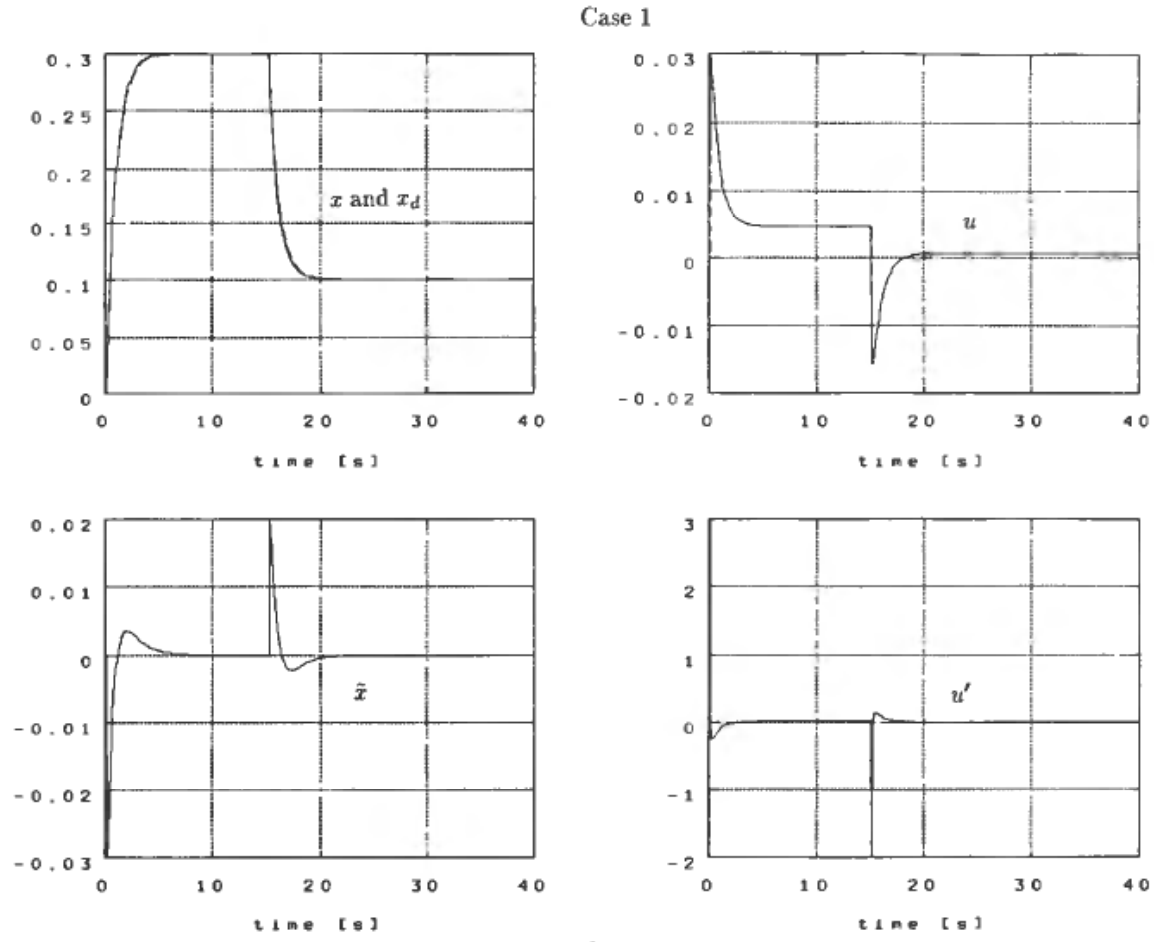

Case 2
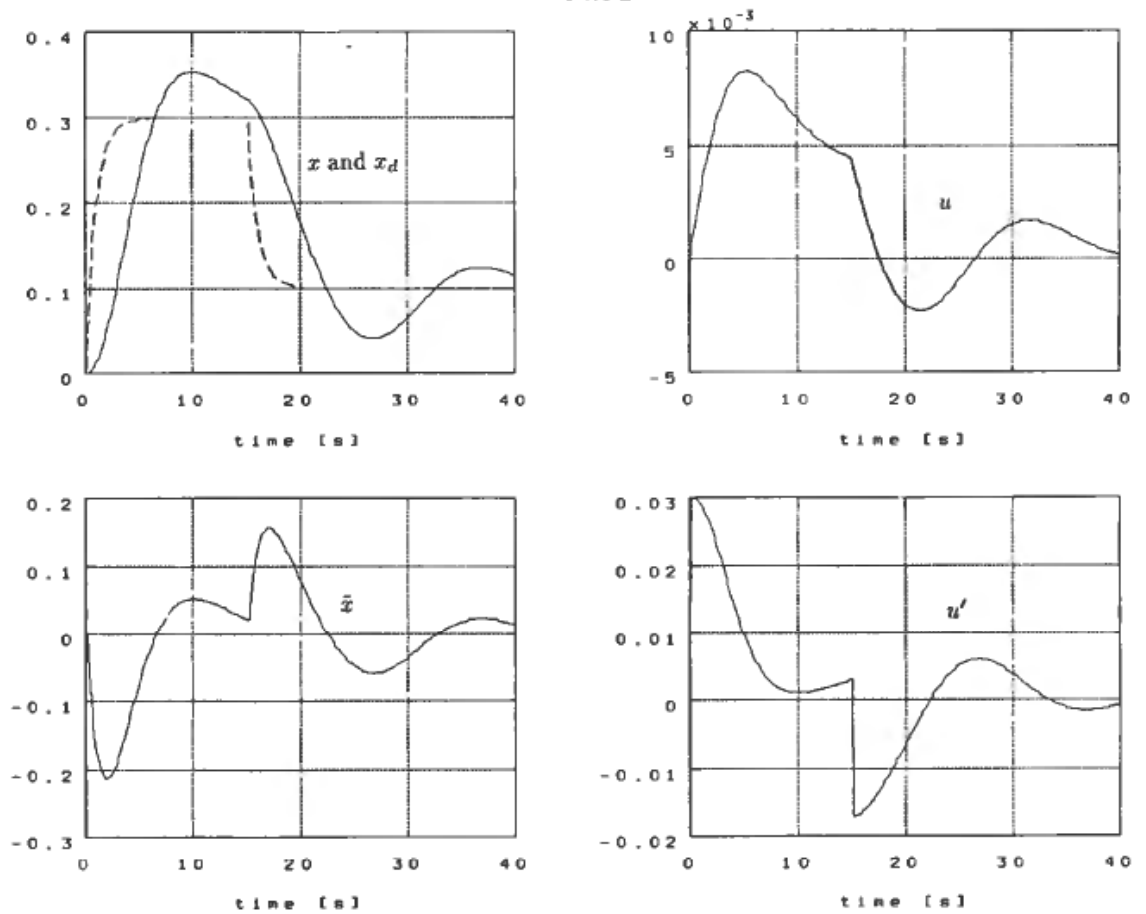

Figure 4. Nominal design $T=10 \mathrm{~s}$. 

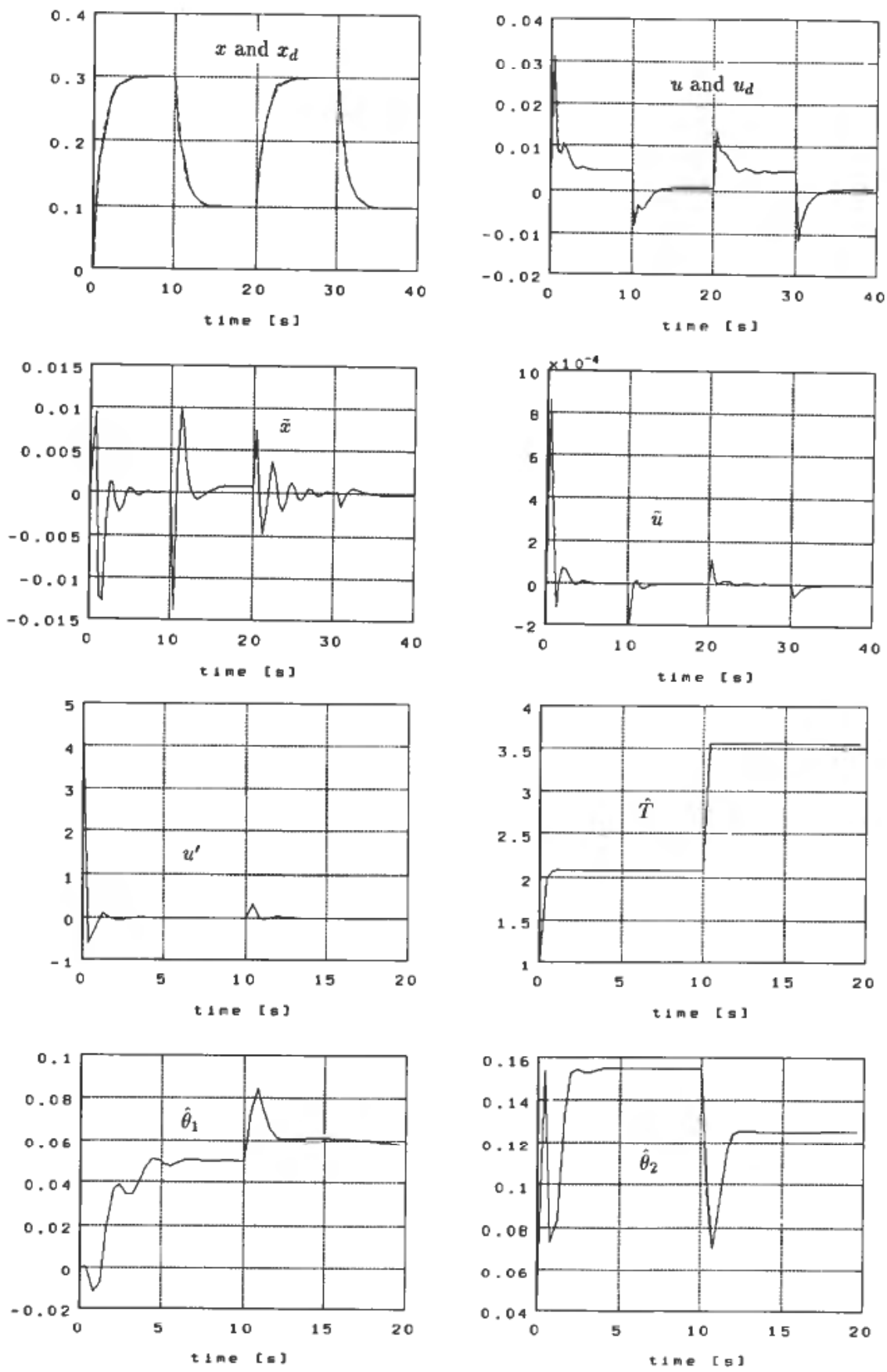

Figure 5. Adaptive feedback linearization: $T=10 \mathrm{~s}$. 
In the case of parametric uncertainties in the model, adaptive feedback linearization should be used. In the last simulation, Fig. 5, we have assumed that both the plant and actuator parameters were unknown. The unknown parameters were initially chosen as: $\hat{\boldsymbol{\theta}}=[0,0.05]^{\mathrm{T}}$ and $\hat{T}=1$ while the true parameters were $\theta=[0 \cdot 05,0 \cdot 1]^{\mathrm{T}}$ and $T=10$. The adaptation gains were selected as: $\gamma_{1}=0.01, \gamma_{2}=0.01$ and $\beta=0.001$ to demonstrate the adaptive case while $\lambda_{u}=300$ and $\lambda_{x}=1$. The relative large value for $\lambda_{u}$ ensures that $r_{u} \approx u$ which is essential for good performance of the outer loop. In the adaptive case, Fig. 5, it is seen that the performance is quite good even for parametric uncertainties. As expected, the unknown parameters do not converge to a global optimum. The scheme still generates parameter values that ensure that the tracking error converges to zero. If parameter convergence is important, the desired trajectory must satisfy certain 'sufficient richness conditions', e.g. see Slotine and $\mathrm{Li}$ (1987). Also note that the inner loop $u$ converges much faster than the outer loop $x$ due to the bandwidth specification: $\lambda_{u}=300 \gg \lambda_{x}=1$. These results illustrate that the actuator time constant like the plant parameters do not have to be perfectly known in advance. The result that the actuator time constant may be estimated and included in the feedback linearization scheme may be quite applicable in control system design.

\section{Conclusions}

An algorithm for adaptive feedback linearization of non-linear systems which includes the systems actuator dynamics has been derived. The control synthesis is divided into two steps: (1) design of a high bandwidth inner loop which decouples the actuator dynamics, and (2) design of an outer loop controller which decouples the plant dynamics. The actuator dynamics does not have to be linear as in this paper. It is straightforward to rederive the scheme for the case when the actuator dynamics are non-linear. In the simulation study, adaptive feedback linearization of a system with significant actuator dynamics was found to yield good performance even for large parametric uncertainties. A generalization to MIMO systems will be essentially mathematical.

\section{Acknowledgments}

The authors are grateful to Svein I. Sagatun for his stimulating discussions. This work was partly sponsored by the Fulbright Foundation, the NATO Science Fellowships Programme and the Royal Norwegian Council for Scientific and Industrial Research through the Center for Robotic Research at the Norwegian Institute of Technology.

\section{REFERENCES}

SASTRY, S. S. and IsIDORI, A. (1989). Adaptive control of linearizable systems. IEEE Trans. Automatic Control, 34, 1123-1131.

Slotine, J. J. E. and COETSEe, J. A. (1986), Adaptive sliding controller synthesis for non-linear systems. Int. Journal of Control, 43, 1631-1651.

Slotine, J. J. E. and Li, W. (1987). Adaptive manipulator control. A Case Study. IEEE Int. Conference on Robotics and Automation, 1987, pp. 1392-1401.

Slorine, J. J. E. and LI, W. (1991). Applied Nonlinear Control (Prentice-Hall, Englewood Cliffs, New Jersey 07632).

YoERGER, D. R., and SLOTINE, J. E. E. (1985). Robust trajectory control of underwater vehicles. IEEE Journal of Oceanic Engineering, 10, 462-470. 